\title{
A Network-Enabled Myoelectric Platform for Prototyping Research Outside of the Lab
}

\author{
Matthew Dyson ${ }^{1}$, Jennifer Olsen ${ }^{1}$ and Sigrid Dupan ${ }^{2}$
}

\begin{abstract}
We present a network-enabled myoelectric platform for performing research outside of the laboratory environment. A low-cost, flexible, modular design based on common Internet of Things connectivity technology allows home-based research to be piloted. An outline of the platform is presented followed by technical results obtained from ten days of home-based tests with three participants. Results show the system enabled collection of close to 12,000 trials during around 28 cumulative hours of use. Home-based testing of multiple participants in parallel offers efficiency gains and provides a intuitive route toward long-term testing of upper-limb prosthetic devices in more naturalistic settings.

Clinical relevance- In-home myoelectric training reduces clinician time. Network-enabled systems with back-end dashboards allow clinicians to monitor patients myoelectric ability over time and will provide a new way of accessing information about how upper-limb prosthetics are commonly used.
\end{abstract}

\section{INTRODUCTION}

Active hand prostheses are most commonly controlled using myoelectric control, i.e. the estimation of user intent from muscle activity in the users residual limb via the electromyogram [1]. A growing body of evidence shows that offline performance in myoelectric control has weak correlation to online control [2], [3]. Accordingly, there is increased demand for user-in-the-loop tests of new and novel systems, necessitating increased participant involvement in system development. Home-based, or outside-of-the-lab, research offers opportunities for more efficient closed-loop data collection in more naturalistic settings.

Closed-loop myoelectrics systems allow users to improve performance. This occurs across various control schemes including direct control [4], pattern recognition [5], [6], [4], [7] and regression [8], [3]. This apparent learning has been investigated in single sessions [8], [3], in multi-day studies [9], [4], [10], [6], [7] and in multi-week studies [5], [10]. There is also a growing body of prosthesis control which uses non-biomimetic mappings between muscle activity and prosthesis grasps [11], [12], [13], [14], [15], [16]. Many of these approaches use motor-learning techniques and consequently performance improves over time [13], [15]. These approaches are not conducive to laboratory testing of naive

*This work was supported by the UK Engineering and Physical Sciences Research Council (EPSRC), (grant number: EP/R511584/1) and by the National Institute for Health Research (NIHR) Devices 4 Dignity (D4D) Starworks (project: STWK-006).

${ }^{1}$ Matthew Dyson and Jennifer Olsen are with Intelligent Sensing Laboratory, Newcastle University, UK matthew.dyson, j.olsen@newcastle.ac.uk

${ }^{2}$ Sigrid Dupan is with the Edinburgh Neuroprosthetics Laboratory, Edinburgh University, UK sigrid.dupanded.ac.uk

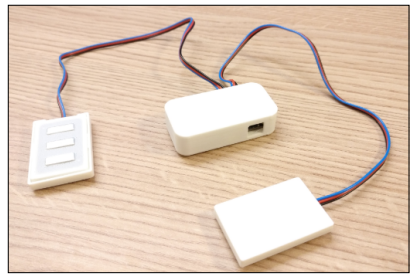

a

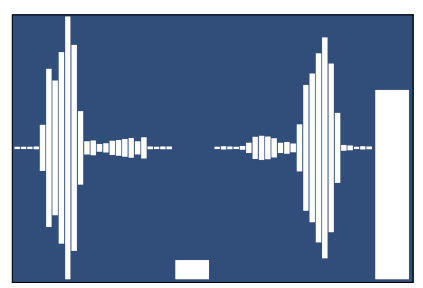

C

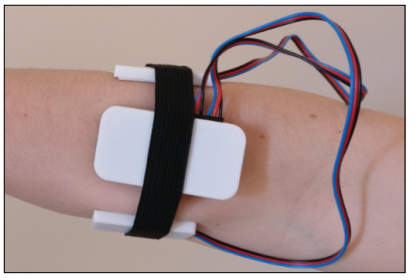

b

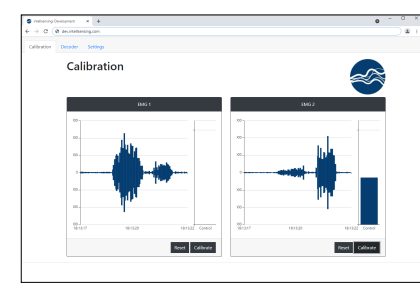

d
Fig. 1. Myoelectric device and sample calibration software. (a) Device in 3D printed housing with two EMG sensors attached. (b) Upper forearm placement with sensors on flexor and extensor. (c) Android interface and (d) browser interface used for calibration showing stylised EMG. Images shows activity associated with wrist extension followed by flexion with representative crosstalk and signal to noise ratio against baseline.

participants because, although performance improvements are observed, predicting when participants will reach proficiency is logistically challenging.

Beyond learning-based prosthesis control, EMG signals acquired during long-term use of pattern recognition systems change over time [17], [7] and error-rates associated with extended use of pattern recognition suggest learning [17]. Whether these factors are linked or causal is unknown. Irrespective, they confound analysis of long term advanced prosthesis use because distinguishing user adaptation from machine adaptation in co-adaptive systems is not trivial [15]. These effects are observed during long-term use of closedloop systems, and, irrespective of control scheme, necessitate large scale data collection for meaningful analyses.

These types of data collection are not practical for most research labs. Instead, it is more pragmatic to use low-cost devices outside of the lab to collect long-term data from multiple participants in parallel. We present a flexible, modular, low cost platform which uses Internet of Things (IoT) connectivity technology [18] and Bluetooth Low Energy (BLE) to achieve this. The platform was developed on the premise that end-to-end systems are necessary to prototype the novel research designs for beyond the lab research. We outline the current state of the platform and preliminary results from ten days of in-home data collection. 


\section{PLATFORM}

The platform comprises a wireless myoelectric device based on a low-cost development board, software for interfacing with hardware appropriate for in-home research and an online server running IoT and storage services.

\section{A. Myoelectric}

The myoelectric device is designed to stream data at low latency. The device firmware implements simple signal processing of EMG data, the abstract decoding scheme described in [1] and a CAN bus for interfacing with upperlimb prosthetics.

1) Device: The prototype has been developed on an Adafruit Feather nRF52 Bluefruit LE. The Feather nRF52 is based on a Nordic Semiconductor nRF52832 generalpurpose multiprotocol system on chip which uses an Arm Cortex M4F processor. The Arm M4F enables floating-point unit hardware acceleration of single precision float calculations. The nRF52832 can perform central or peripheral mode BLE, can concurrently run multiple BLE protocols and transmit at a bit rate of 2 Mbps. Device communication occurs over BLE.

2) Sensors: Electromyography signal acquisition is performed using modified OYMotion Analog EMG Sensors. Modifications were made to reduce sensor noise and the physical profile. The OYMotion sensor comprises two boards connected by a $3.5 \mathrm{~mm}$ audio cable. The $3.5 \mathrm{~mm}$ PCB mount stereo jack sockets are removed as are the JST connectors. All contacts are then directly soldered and the PCBs are vertically stacked. Inertial data is acquired using a Bosch BNO055 Intelligent 9-Axis Absolute Sensor which outputs fused sensor data in quaternion form. Typically two channels of EMG are sampled at $500 \mathrm{~Hz}$ and inertial data is sampled at $20 \mathrm{~Hz}$.

3) CAN bus: The myoelectric can interface with upperlimb prostheses via a CAN bus module. A low power Microchip Technology MCP2515 Controller Area Network Controller can be powered by the $3.3 \mathrm{~V}$ Adafruit Feather. The MCP2515 provides CAN V2 at $1 \mathrm{Mb} / \mathrm{s}$. The interface has been tested with a Touch Bionics (Össur) RoboLimb.

\section{B. Local devices}

Local devices communicate with the myoelectric device using BLE and can interact with a remote server. In principle, each device can fulfill any purpose. In practice devices currently have relatively specialist roles. The device is shown in Figures 1a and 1b.

1) Computer: Desktop computers have been used for in-home experiments. The device can be interfaced using Python or C\#. The AxoPy Python library is used for myoelectric computer interface experiments [19]. The Unity development engine is used for myogame development [20].

2) Mobile: Mobile applications are developed in C\#. The Unity development engine is used for rapid prototyping of software for configuring and calibrating the myoelectric device. An example of an EMG calibration system running on a mobile phone is shown in Figure 1c.

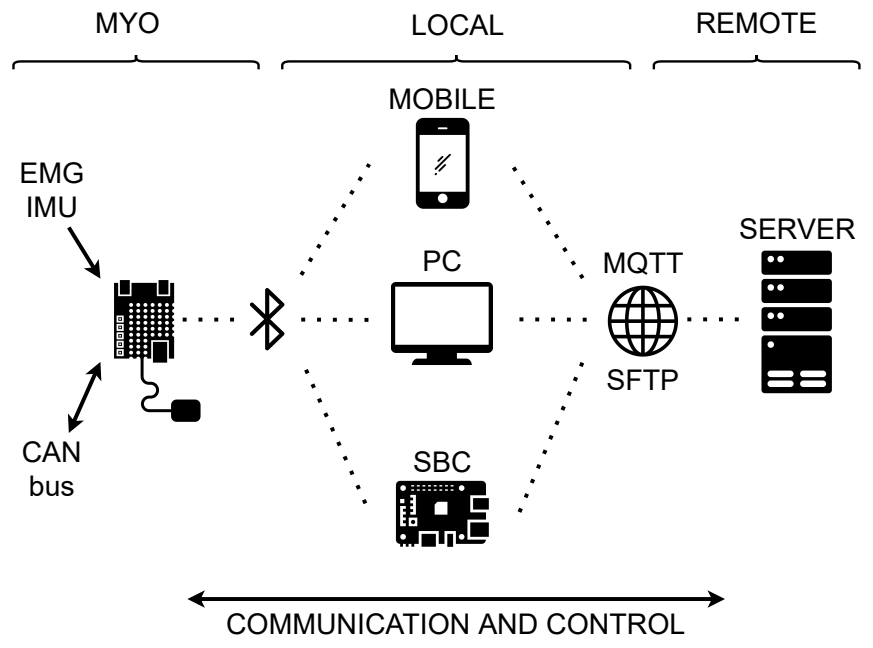

Fig. 2. Overview of platform. Myoelectric with EMG and IMU sensors and optional CAN Bus interface communicates with local devices over BLE. General purpose local devices can communicate with a remote server using MQTT and SFTP protocols. The remote server provides a range of online IoT services. Communication and control is bidirectional between the myoelectric and all other devices on the platform.

3) Single Board Computer: The Raspberry Pi is a low power, low cost, single board computer with a small form factor and are used for passive monitoring of the myoelectric device, bridging of the device to the internet and local provision of non-CPU intensive services.

\section{Remote server}

A remote backend server provides services which allow data to be collected outside of the lab. Services are compartmentalised for security. The core services used are:

1) Message Queuing Telemetry Transport: Message Queuing Telemetry Transport (MQTT) is a standard IoT messaging protocol which uses a publish/subscribe transport. A secure MQTT broker runs on the server. The MQTT broker communicates over Websockets and data is encrypted using Transport Layer Security (TLS).

2) Secure File Transfer Protocol: Secure Shell (SSH) File Transfer Protocol (SFTP) is a web file transfer protocol. The SFTP protocol enables remote file management capabilities and resumption in the case of transfer failure. SFTP authentication is performed using public-key cryptography.

3) Node-RED: Node-RED is a flow-based development tool designed for edge-computing by IBM research. The technology stack behind Node-RED is a node.js Javascript runtime and Javascript. A browser interface is used for flow-based programming. Node-RED exposes a range of open source components which enable rapid prototyping and development of online IoT services. An example of a NodeRED browser-based EMG calibration system is shown in Figure 1d.

An overview of the platform is shown in Figure 2. An example experiment which uses the platform to train participants in EMG control is described in the next section. 


\section{EXAMPLE EXPERIMENT}

\section{A. Participants}

Three participants ( 1 female, 2 male) were recruited. All of the participants were able-bodied, free from any neurological or motor disorder, and gave written informed consent. Ethical approval was granted by the local committee at Newcastle University (Ref: 17-NAZ-056).

\section{B. Experimental setup}

Three participants performed ten days of myoelectric training in the home. Two EMG channels were used, EMG data was streamed over BLE to a PC running the AxoPy Python library for real-time myoelectric experiments. Participants performed a version of the abstract decoding protocol described in [13], [15] which had been modified to reduce any reliance upon visual feedback. Calibration routines were provided which participants used to normalise the EMG activity they used in task control. Calibration of EMG activity followed the procedure used in previous abstract decoding experiments [13], [15].

Each participant did one recording session per day. Participants performed experimental runs comprised of 60 trials in their own time. At the end of each run the resulting experimental data - control signal data, configuration values, and individual trial scores - were compressed and transmitted to the remote server using SFTP. The MQTT service maintained a log of participants performance over time. At the end of each experimental run, participants were presented with a graph showing their score over time along with a scoreboard listing the maximum score achieved by each of the participants involved. Further details regarding the experimental protocol used for the first five days of experiments, and preliminary results obtained, can be found in [21].

\section{Results}

An overview of use over ten days is shown in Figure 3. Trial counts were calculated based on all data transmitted to the server, inclusive of any false start experimental runs. Figure $3 \mathrm{a}$ shows total trials acquired over ten days. During the first five days 5480 trials were recorded. This includes additional evaluation trial data not reported in [21]. In total 11611 trials were recorded over ten days. Figure 3b shows the total hours of use. Hours of use was measured as the time between starting training and ending training during sessions. In total there were close to 28 hours of use. Cumulative hours of data recorded are shown in Figure 3c, these values were calculated based on the time stamps of files acquired. Close to 20 hours of experimental data were recorded over ten days.

A brief breakdown of data by participants is shown in Figure 4. Figure 4a shows the median recording session time for each participant. There was no significant difference in median session times between participants (Kruskal-Wallis, $d f=2, x^{2}=4.35, p=0.1134$ ). Total trial counts per participant are shown in Figure 4b. All participants recorded over 3000 trials, participants two and three recording over 4000 trials.
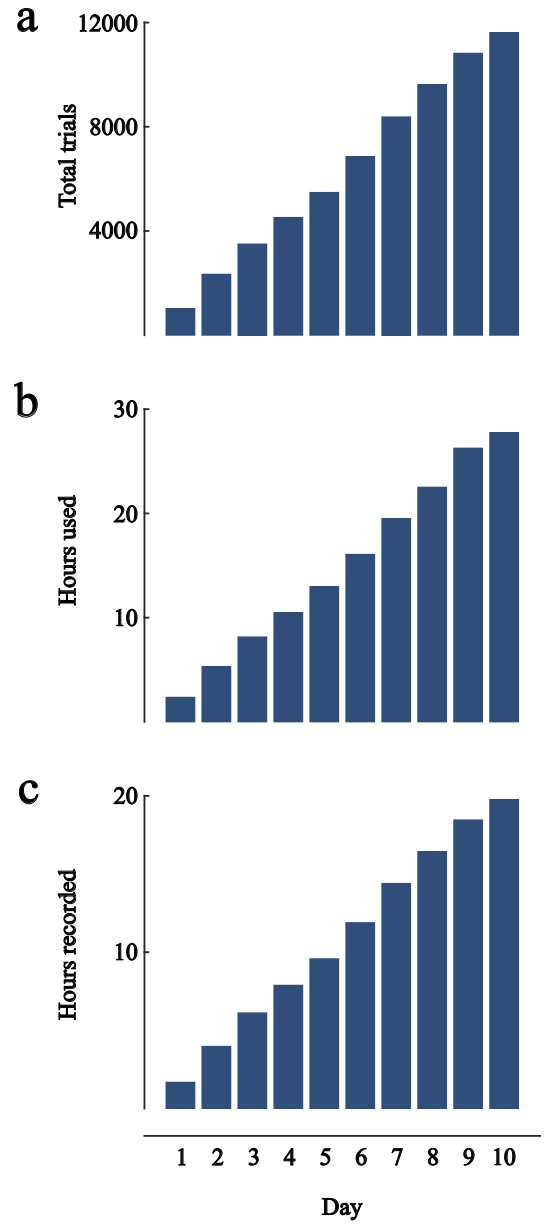

Fig. 3. Overview of platform use over ten days. Sub-plots share the same horizontal axis. (a) Cumulative experimental trials recorded and sent to the remote server. (b) Cumulative hours used, measured as initiating a recording session to completing one, ignoring calibration. (c) Cumulative hours of data recorded, measured as time series of experimental data recorded.
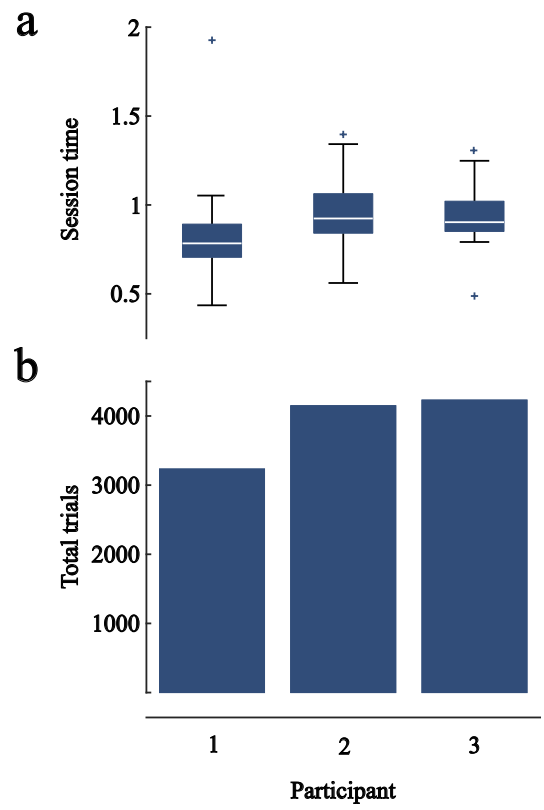

Fig. 4. Overview of individual participant use of the platform. Sub-plots all share the same horizontal axis. (a) Median session length for each participant. (b) Total trials for each participant. 


\section{CONCLUSIONS}

We have demonstrated that a network-enabled myoelectric system can be used to perform a motor-learning based experiment in a home setting. With a relatively short time commitment per day all participants managed to perform somewhere between 1500 and 2000 trials per week. For myoelectric approaches which induce behavioural changes via brain plasticity, these methods present significant advantages by providing a method whereby the required number of repetitions to induce long-term changes may be reached without a proportional commitment from experimental operators. Although the results presented were for a experiment presented on a desktop computer, the same process could be achieved on a mobile device in any location.

What is arguably missed in technical or experimental reports of home-based myoelectric training is the comparative convenience relative to laboratory-based testing. Although there is an incurred cost in ensuring the hardware and software used are sufficiently robust for use outside of the lab, it is likely that the resources created can be reused. The returns for the experimental operator are a passive data collection exercise which can easily be scaled to multiple parallel participants. Participant travel time can be significantly reduced and scheduling restraints are removed. Near real-time connectivity ensures the experimental operator can still diagnose technical problems.

The weak correlation observed between offline myoelectric performance and control [2] is both a cause, and a consequence, of upper-limb prosthetics failure to transition published gains in laboratory research to real-world use. Few research groups progress theoretical work to the multidisciplinary clinical work required to test applied real-time control outside of the lab [5]. As such, a reliance on laboratory-based development, often using offline data, means critical factors such as a changing input control signal [17], [7], and more generally the influence of time on real-time performance [10], have only begun to be addressed recently.

Platforms for performing research outside of the laboratory can provide methods for addressing these challenges and those that follow. With respect to data collection, both passive tracking of continuous naturalistic movements and active prompting of labelled posture or activity data can be achieved using a streaming platform with bidirectional connectivity. During long-term data collection the approach enables use of streaming data algorithms, significantly reducing the bottlenecks associated with treating data collection and analysis as distinct research phases. Finally, when applied to functional prosthesis use and control, IoT connectivity will allow a range of alternative development, test, monitoring and experimental approaches.

Internet enabled outside-of-the-lab testing can be more convenient and efficient than traditional methods. As such, we anticipate it will allow for greater user involvement in future upper-limb prosthetics research, with participants involved earlier, at more stages of the process, in greater numbers, and at more physical locations.

\section{REFERENCES}

[1] G. Hefftner and G. Jaros, "The electromyogram EMG as a control signal for functional neuromuscular stimulation-part II: practical demonstration of the EMG signature discrimination system," IEEE Trans on Biomedical Engineering, vol. 35, no. 4, pp. 238-242, 1988.

[2] I. Vujaklija, A. D. Roche, T. Hasenoehrl, A. Sturma, S. Amsuess, D. Farina, and O. C. Aszmann, "Translating research on myoelectric control into clinics-are the performance assessment methods adequate?" Frontiers in Neurorobotics, vol. 11, p. 7, 2017.

[3] A. Gigli, A. Gijsberts, and C. Castellini, "The Merits of Dynamic Data Acquisition for Realistic Myocontrol," Frontiers in Bioengineering and Biotechnology, vol. 8, no. April, 2020.

[4] H. Bouwsema, C. K. van der Sluis, and R. M. Bongers, "Changes in performance over time while learning to use a myoelectric prosthesis," Journal of NeuroEngineering and Rehabilitation, vol. 11, no. 16, 2014.

[5] L. J. Hargrove, L. A. Miller, K. Turner, and T. A. Kuiken, "Myoelectric pattern recognition outperforms direct control for transhumeral amputees with targeted muscle reinnervation: A randomized clinical trial," Scientific Reports, vol. 7, p. 13840, 2017.

[6] X. Sheng, B. Lv, W. Guo, and X. Zhu, "Common spatial-spectral analysis of EMG signals for multiday and multiuser myoelectric interface," Biomedical Signal Processing and Control, vol. 53, 2019.

[7] M. B. Kristoffersen, A. W. Franzke, C. K. van der Sluis, A. Murgia, and R. M. Bongers, "The effect of feedback during training sessions on learning pattern-recognition-based prosthesis control," IEEE Transactions on Neural Systems and Rehabilitation Engineering, vol. 27, no. 10, pp. 2087-2096, 2019.

[8] M. Couraud, D. Cattaert, F. Paclet, P. Y. Oudeyer, and A. de Rugy, "Model and experiments to optimize co-adaptation in a simplified myoelectric control system," Journal of Neural Engineering, vol. 15, no. 2, p. $026006,2018$.

[9] G. C. Matrone, C. Cipriani, M. Chiara Carrozza, and G. Magenes, "Real-time myoelectric control of a multi-fingered hand prosthesis using principal components analysis," Journal of NeuroEngineering and Rehabilitation, vol. 9, no. 40, 2012.

[10] A. Waris, I. Mendez, K. Englehart, W. Jensen, and E. N. Kamavuako, "On the robustness of real-time myoelectric control investigations: A multiday Fitts' law approach," Journal of Neural Engineering, vol. 16, no. 2, 2019.

[11] S. M. Radhakrishnan, S. N. Baker, and A. Jackson, "Learning a novel myoelectric-controlled interface task," Journal of Neurophysiology, vol. 100, no. 4, pp. 2397-2408, 2008.

[12] J. L. Segil and R. F. Weir, "Novel postural control algorithm for control of multifunctional myoelectric prosthetic hands," Journal of Rehabilitation Research and Development, vol. 52, no. 4, pp. 449466, 2015.

[13] M. Dyson, J. Barnes, and K. Nazarpour, "Myoelectric control with abstract decoders," Journal of Neural Engineering, vol. 15, no. 5, p. $056003,2018$.

[14] C. W. Antuvan, "Decoding human motion intention using myoelectric signals for assistive technologies," Ph.D. dissertation, Nanyang Technological University, Singapore, 42019.

[15] M. Dyson, S. Dupan, H. Jones, and K. Nazarpour, "Learning, generalization, and scalability of abstract myoelectric control," IEEE Transactions on Neural Systems and Rehabilitation Engineering, vol. 28, no. 7, pp. 1539-1547, 2020.

[16] J. Segil, R. Kaliki, J. Uellendahl, and R. F. Weir, "A myoelectric postural control algorithm for persons with transradial amputation: A consideration of clinical readiness," IEEE Robotics and Automation Magazine, 2020.

[17] J. He, D. Zhang, N. Jiang, X. Sheng, D. Farina, and X. Zhu, "User adaptation in long-term, open-loop myoelectric training: implications for EMG pattern recognition in prosthesis control," Journal of Neural Engineering, vol. 12, no. 4, p. 046005, 2015.

[18] OpenJS Foundation, "Node-RED: Low-code programming for eventdriven applications," https://nodered.org, 2021.

[19] K. R. Lyons and B. W. L. Margolis, "AxoPy: A Python library for implementing human-computer interface experiments," Journal of Open Source Software, vol. 4, no. 34, p. 1191, 2019.

[20] Unity Technologies, "Unity real-time development platform | 3D, 2D, VR \& AR engine," https://unity.com, 2021.

[21] S. Stuttaford, S. Dupan, K. Nazarpour, and M. Dyson, "Long-term myoelectric training with delayed feedback in the home environment," in Proc. of the IEEE Engineering in Medicine and Biology Annual Conference. IEEE, October 2021 (Submitted). 